\title{
An Experimental Model of Postnatal Jaundice in the Suckling Rat Suppression of Induced Hyperbilirubinemia by Sn-Protoporphyrin
}

George S. Drummond and Attallah Kappas

The Rockefeller University Hospital, New York 10021

bstract. A model of experimental postnatal hyperbilirubinemia in the rat has been developed utilizing the heme precursor $\delta$-aminolevulinic acid (ALA) to produce jaundice during a selective time period after birth. This time period is defined as that between $7 \mathrm{~d}$ postnatally, when the initial postpartum alterations of serum bilirubin and heme metabolism in the neonate have subsided, and $21 \mathrm{~d}$, when the hepatic conjugation mechanism for the bile pigment appears fully developed.

Administration of ALA in this time period led to a rapid, consistent, and significant dose-dependent increase in serum bilirubin levels in the newborn animals. Heme administration produced a qualitatively similar but enhanced effect. Both compounds, in addition, induced a dose-dependent increase in hepatic heme oxygenase activity concomitant with the increase in serum bilirubin levels. Neither compound increased serum bilirubin levels significantly when administered at or after $21 \mathrm{~d}$ postnatally. Administration of the synthetic metalloporphyrin, Sn-protoporphyrin, to ALA-treated neonates resulted in a dose-dependent decrease in serum bilirubin levels and hepatic heme oxygenase activity. $\mathrm{Mn}$ - and $\mathrm{Zn}$-protoporphyrin in comparable doses did not significantly inhibit ALA-induced hyperbilirubinemia. Sn-protoporphyrin also inhibited the hyperbilirubinemia produced by heme in the suckling animals.

ALA administration to newborn rats during the specific postnatal period described provides a simple and convenient model of experimental jaundice in the developing neonate which permits an examination of the potential ability of synthetic metalloporphyrins or other compounds to suppress induced hyperbilirubinemia in

Received for publication 19 December 1983 and in revised form 29 March 1984.

J. Clin. Invest.

(c) The American Society for Clinical Investigation, Inc. 0021-9738/84/07/0142/08 \$1.00

Volume 74 , July $1984,142-149$ the newborn animal. The ability to induce a consistent and significant degree of jaundice in the postnatal rat by the method described may also be useful for other types of studies concerned with the biological disposition and effects of endogenously formed bilirubin in the neonate.

The results of this study confirm in another model system the potent ability of Sn-protoporphyrin to suppress jaundice in the neonate, and suggest that suppression of heme oxidation by synthetic heme analogues may represent a useful therapeutic approach to the problem of severe hyperbilirubinemia in human premature newborn.

\section{Introduction}

Hyperbilirubinemia in the newborn resulting in part from exaggerated erythrocyte destruction, enhanced rates of tissue heme oxidation, and immaturity of the hepatic bilirubin conjugation mechanism is common in human premature newborn (1). Therapeutic approaches to this problem presently involve methods for diminishing plasma bilirubin levels by attempting to dispose of the bile pigment after it has been produced in the heme degradation process. Recent studies from this laboratory $(2,3)$ have demonstrated that it is also possible to suppress excessive plasma bilirubin levels in the animal neonate by the administration of a synthetic metalloporphyrin which acts as a potent competitive inhibitor of heme oxygenase, the rate-limiting enzyme in the catabolism of heme to bilirubin. In this approach, plasma levels of bilirubin are diminished by potently inhibiting production of the bile pigment rather than by attempting to dispose of bilirubin after it has been produced in the heme oxidation sequence.

The synthetic metalloporphyrin Sn-(tin)protoporphyrin, which has a very low inhibition constant $\left(K_{\mathrm{i}}\right)$ for microsomal heme oxygenase in animal (2) and human tissues (3) as well as for the homogenously purified enzyme (4), has proved especially effective in suppressing hyperbilirubinemia in the neonatal rat $(2,3)$, and also, in adult mice with severe hereditary hemolytic anemia (5). This action of the metalloporphyrin has been confirmed in the nonhuman primate, including the Rhesus neonate $(6,7) . \mathrm{Cr}$ (chromium)-protoporphyrin, also, has the capacity to suppress hyperbilirubinemia in the rat neonate (8). The results of these studies confirm the suggestion made by this laboratory 
in 1975 (9) that a therapeutic approach to the problem of newborn jaundice might be developed by considering means for regulating heme degradation at a low rate in early neonatal life.

To further explore the potential of Sn-protoporphyrin and other synthetic metalloporphyrins for suppressing postnatal jaundice, we have developed a model system for examining the effects of these compounds on the hyperbilirubinemia which can be experimentally induced in the suckling rat. In this report, we demonstrate that administration of heme or the heme precursor, $\delta$-aminolevulinate (ALA), ${ }^{\prime}$ to rats during a selective time period postnatally produces a rapid, consistent, and significant increase in the level of serum bilirubin and that the administration of Sn-protoporphyrin concurrently with these agents significantly reduces the increase in serum bilirubin levels which they elicit. The model of experimental postnatal jaundice which is described permits induction of significant degrees of hyperbilirubinemia in the postnatal rat and has the advantage that it utilizes a convenient laboratory animal. In addition to its use for the experiments described in this report, it may also prove applicable to other types of studies concerned with the biological disposition and effects of endogenously formed bilirubin in the newborn.

\section{Methods}

Materials. Male (175-225 g) and 15-d pregnant female Sprague Dawley rats supplied by Taconic Farms, Inc. (Germantown, NY) and Holtzman Co. (Madison, WI), respectively, were used throughout this study. Pregnancy was synchronized in females so that sufficient numbers of newborn could be studied within the same postnatal time period. Metalloporphyrins were purchased from Porphyrin Products (Logan, UT) and ALA was purchased from Sigma Chemical Co. (St. Louis, MO). All other chemicals were of the highest grade obtainable from either Sigma Chemical Co. or Fisher Scientific Co. (Pittsburg, PA). ALA hydrochloride$\delta\left[4-{ }^{14} \mathrm{C}\right](53.2 \mathrm{mCi} / \mathrm{mmol}$ in $0.1 \mathrm{M} \mathrm{HCl})$ was purchased from New England Nuclear (Boston, MA).

Animal treatment. ALA was administered intraperitoneally at various doses up to and including $100 \mu \mathrm{mol} / 100 \mathrm{~g}$ body weight (bw) 24,20 , and $16 \mathrm{~h}$ prior to sacrifice unless otherwise indicated. Metalloporphyrins including $\mathrm{Fe}$ (iron)-protoporphyrin (heme) were administered subcutaneously in the nuchal region at various doses up to and including 100 $\mu \mathrm{mol} / \mathrm{kg}$ bw $24 \mathrm{~h}$ prior to sacrifice. To prepare metalloporphyrin solutions, the metalloporphyrin was dissolved in a small volume of $0.5 \mathrm{M} \mathrm{NaOH}$ $(0.2 \mathrm{ml} / 1.0 \mathrm{ml}$ of final volume of metalloporphyrin solution) adjusted to $\mathrm{pH} 7.4$ with $1 \mathrm{M} \mathrm{HCl}$, and made up to final volume with $0.9 \% \mathrm{NaCl}$. Control animals were administered an equivalent volume of $0.9 \% \mathrm{NaCl}$. The final volume of each injection was $0.1 \mathrm{ml}$ in neonates up to 14-dold. Neonates remained with their mothers during the course of the treatments. Neonates were weaned when 28-d-old. Groups of neonates (6-30, depending on age) were killed at the times indicated. Animals were housed in The Rockefeller University Laboratory Animal Research Center and were maintained in a controlled environment with a $12 / 12$ h light/dark cycle.

1. Abbreviations used in this paper: ALA, $\delta$-aminolevulinic acid; bw, body weight.
Tissue preparation. Livers were perfused in situ with ice cold $0.9 \%$ $\mathrm{NaCl}$ and homogenized in 3 vol of $0.1 \mathrm{M}$ potassium phosphate buffer, $\mathrm{pH} 7.4$, containing $0.25 \mathrm{M}$ sucrose, and the microsomal fractions were prepared as previously described for the determination of heme oxygenase activity (2). Spleen microsomal fractions were prepared in an identical manner. The cytosolic fraction from control animals served as the source of biliverdin reductase.

Assays. The activity of heme oxygenase was assayed as previously described (2). Bilirubin formation was calculated by using an absorption coefficient of $40 \mathrm{mM}^{-1} \mathrm{~cm}^{-1}$ between 464 and $530 \mathrm{~nm}$. Total plasma bilirubin was estimated fluorometrically by the method of Roth (10) using an Hitachi MPF III fluorescence spectrophotometer equipped with an R928 photomultiplier tube; the variation in replicate samples was $<5 \%$. Metalloporphyrins did not interfere with the determination of serum bilirubin in this assay. The conversion of $\left[{ }^{14} \mathrm{C}\right] \mathrm{ALA}$ to heme was measured by the method of Healey et al. (11). Protein content was determined by the method of Lowry et al. (12) using crystalline bovine serum albumin as standard. The data were analyzed by the standard $t$ test and the value $P<0.05$ was regarded as significant.

\section{Results}

Effect of ALA and heme on serum bilirubin levels in 7-d-old neonates. Previous studies demonstrated that ALA administered in three divided doses to starved 6-d-old neonates markedly increased serum bilirubin levels when measured $24 \mathrm{~h}$ later (13). We reexamined this effect of ALA in suckling 7-d-old neonates to determine whether milk deprivation of the newborn animal was necessary for this hyperbilirubinemia to occur. Increasing amounts of ALA administered to the neonates in three divided doses $(25-100 \mu \mathrm{mol} / 100 \mathrm{~g}$ bw each) produced a significant dosedependent increase in serum bilirubin levels measured $24 \mathrm{~h}$ after the initial administration of ALA (Fig. 1); this increase was less than that observed in starved neonates (13) but was nevertheless consistent and substantial. Serum bilirubin levels had returned to near-normal levels by $48 \mathrm{~h}$ (results not shown). Associated with the increase in serum bilirubin levels after ALA administration was a dose-dependent increase in hepatic heme oxygenase activity (Fig. 1). No increase in splenic heme oxygenase was observed after ALA administration (results not

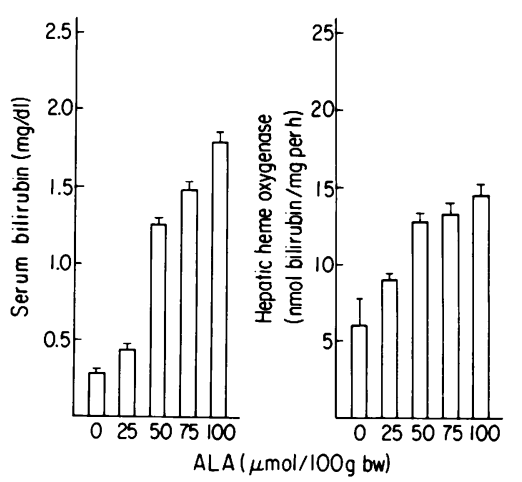

Figure 1. Dose response of ALA on serum bilirubin levels and hepatic heme oxygenase activity in 7-d-old neonates. ALA was administered intraperitoneally to 6-dold suckling neonates in three separate doses at the concentrations indicated 24,20 , and $16 \mathrm{~h}$ prior to sacrifice. Heme oxygenase activity and serum bilirubin levels were assayed as described in Methods. A minimum of 24 neonates were used in each group. 
shown). Based on these results, a dose of $3 \times 50 \mu \mathrm{mol} / 100 \mathrm{~g}$ bw of ALA, which produced an $\sim$ fivefold increase in serum bilirubin, was used in subsequent experiments.

A similar dose-dependent increase in serum bilirubin levels was noted when heme was administered in two divided doses (Fig. 2). The increase in serum bilirubin levels was three- to fourfold higher with heme administration when approximately equimolar amounts of heme equivalents (heme, $2 \times 10 \mu \mathrm{mol} /$ $100 \mathrm{~g} \mathrm{bw})$ or ALA $(3 \times 50 \mu \mathrm{mol} / 100 \mathrm{~g} \mathrm{bw})$ were administered to neonates. However, heme administration had a detrimental effect on the behavioral and suckling patterns of the neonates and the greater hyperbilirubinemia produced by the compound is attributed in part to interruption of feeding.

Comparison of the effects of ALA and heme on serum bilirubin levels and hepatic heme oxygenase activity in adult rats. ALA was administered to adult rats (175-225 g) either in three divided doses of $50 \mu \mathrm{mol} / 100 \mathrm{~g}$ bw or a single dose of 150 $\mu \mathrm{mol} / 100 \mathrm{~g} \mathrm{bw}$, and the levels of serum bilirubin and hepatic heme oxygenase activity were examined $24 \mathrm{~h}$ after the initial administration of ALA. No increase in serum bilirubin was noted after either method of ALA treatment despite a significant $(\sim$ fourfold) increase in hepatic heme oxygenase activity (Table I). This lack of effect of administered ALA on serum bilirubin levels in adult rats is considered to reflect the efficiency of the hepatic glucuronyltransferase system for bilirubin in the mature animal. Heme $(2 \times 10 \mu \mathrm{mol} / 100 \mathrm{~g} \mathrm{bw})$ administered to adult rats produced a greater ( $\sim$ ninefold) increase in hepatic heme oxygenase activity but serum bilirubin levels increased only minimally. Thus, neither ALA nor heme produced significant elevations of serum bilirubin levels in mature animals.

Effect of $A L A$ and heme on serum bilirubin levels and hepatic heme oxygenase activity with age in the neonate. ALA ( $50 \mu \mathrm{mol} /$ $100 \mathrm{~g} \mathrm{bw}$ ) was administered in three separate doses to suckling neonates at 24,20 , and $16 \mathrm{~h}$ prior to sacrifice when serum bilirubin levels and hepatic heme oxygenase activity were examined. ALA administration did not commence until day 6 at which time the high levels of hepatic heme oxygenase that occur immediately after birth had begun to decline towards normal

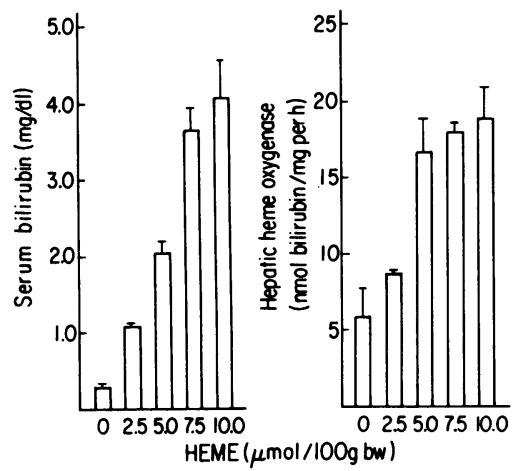

Figure 2. Dose response of heme on serum bilirubin levels and hepatic heme oxygenase activity in 7-d-old neonates. Heme was administered subcutaneously to 6 -dold suckling neonates in the nuchal region in two separate doses at the concentrations indicated 24 and $16 \mathrm{~h}$ prior to sacrifice. Heme oxygenase activity and

serum bilirubin levels were assayed as described in Methods. A minimum of 24 neonates were used in each group.
Table I. Effect of ALA and Heme on Hepatic Heme Oxygenase Activity and Serum Bilirubin Levels in Adult Male Rats

\begin{tabular}{lcl}
\hline Treatment & $\begin{array}{l}\text { Hepatic heme } \\
\text { oxygenase }\end{array}$ & $\begin{array}{l}\text { Serum } \\
\text { bilirubin }\end{array}$ \\
\hline & $n m o l$ bilirubin $/ \mathrm{mg} / \mathrm{h}$ & $\mathrm{mg} / \mathrm{dl}$ \\
Saline & $2.77 \pm 0.21$ & $0.16 \pm 0.01$ \\
ALA $(3 \times 50 \mu \mathrm{mol} / 100 \mathrm{~g} \mathrm{bw})^{*}$ & $10.87 \pm 0.34$ & $0.18 \pm 0.01$ \\
ALA $(150 \mu \mathrm{mol} / 100 \mathrm{~g} \mathrm{bw}) \ddagger$ & $9.79 \pm 1.39$ & $0.18 \pm 0.02$ \\
Heme $(2 \times 10 \mu \mathrm{mol} / 100 \mathrm{~g} \mathrm{bw}) \S$ & $21.65 \pm 1.79$ & $0.31 \pm 0.03$
\end{tabular}

ALA administered intraperitoneally.

* 24,20 , and $16 \mathrm{~h}$ prior to sacrifice.

$\ddagger 24 \mathrm{~h}$ prior to sacrifice.

$\S$ Heme administered subcutaneously 24 and $16 \mathrm{~h}$ prior to sacrifice.

Data are expressed as the mean $\pm \mathrm{SE}$ of a minimum of three determinations.

adult levels $(2,9,14)$. Marked increases in serum bilirubin levels were noted with ALA treatment at 7, 10, and $14 \mathrm{~d}$ after birth; by $21 \mathrm{~d}$, however, when the neonates weighed $\sim 48 \mathrm{~g}$, ALA administration produced no significant increase in serum bilirubin levels in treated animals when compared with levels in control animals and this pattern continued through the $42 \mathrm{~d}$ of study at which time the animals weighed $\sim 175 \mathrm{~g}$ (Fig. 3). Hepatic heme oxygenase activity followed the well-documented developmental pattern of being high at birth and for several days thereafter, before gradually declining to adult levels between
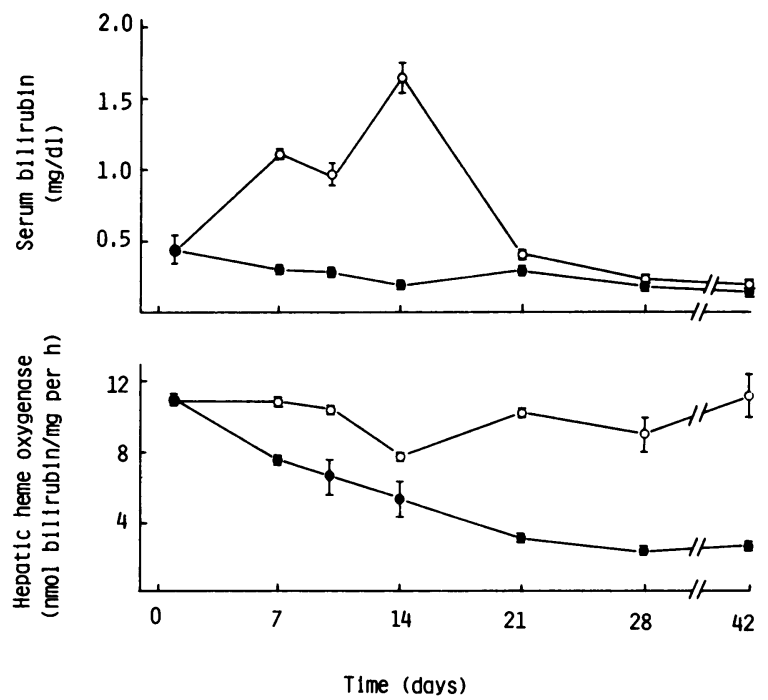

Figure 3. Effect of ALA on serum bilirubin levels and hepatic heme oxygenase in the developing neonate. ALA $(50 \mu \mathrm{mol} / 100 \mathrm{~g}$ bw) was administered intraperitoneally to neonates in three separate doses 24 , 20 , and $16 \mathrm{~h}$ prior to sacrifice at the times indicated. Heme oxygenase activity and serum bilirubin levels were assayed as described in Methods. A minimum of 24 neonates were used at each time point. Control, •; ALA-treated, O. 
21-28 d after birth $(2,9,14)$. ALA administration produced a slight induction response $(44,57$, and $45 \%$ ) in hepatic heme oxygenase activity at days 7,10 , and 14 , respectively; from day 21 on, ALA treatment produced substantial induction responses of hepatic heme oxygenase activity which were comparable with those observed in adult rats (Table I). ALA administration did not affect suckling behavior in the animals or the normal developmental pattern of increasing splenic heme oxygenase activity $(2,9,14,15)$ during the first 2 wk after birth (results not shown).

Heme $(2 \times 10 \mu \mathrm{mol} / 100 \mathrm{~g} \mathrm{bw})$ was administered to suckling neonates 24 and $16 \mathrm{~h}$ prior to sacrifice when serum bilirubin levels and hepatic heme oxygenase activity were determined. Administration of this natural metalloporphyrin resulted in a substantial increase in serum bilirubin levels at days 7-21 after birth when compared with bilirubin levels in control animals or in ALA-treated animals (Table II); after day 21 and through day 42 of the study, serum bilirubin levels in heme-treated animals had declined to levels close to those observed in the untreated group (Table II) indicating that the hyperbilirubinemic response to heme, like that to ALA, was limited to the postnatal period before the hepatic glucuronyl transferase for bilirubin is fully matured $(16,17)$. Heme administration also resulted in a significant increase in hepatic heme oxygenase activity at all time points studied. The pattern of induced heme oxygenase activity was similar to that observed after ALA administration, but was significantly greater in degree (Table II). However, as noted earlier, heme administration in this experiment consistently produced adverse effects on the normal suckling and feeding behavior of the neonates. This was confirmed by smaller volumes of gastric milk content and smaller body weight gains in heme-treated as compared with normal animals. Because of these detrimental effects of the compound in the neonates, the remainder of the studies described were conducted in 7-d-old animals utilizing administered ALA to produce hyperbilirubinemia.

Effect of ALA administration on serum bilirubin levels and hepatic heme oxygenase with time in 7-d-old neonates. ALA $(50 \mu \mathrm{mol} / 100 \mathrm{~g} \mathrm{bw})$ was administered to neonates at the beginning ( 0 ) and 4 and $8 \mathrm{~h}$ later in the experiment shown in Fig.

Table II. Effect of Heme on Serum Bilirubin Levels and Hepatic Heme Oxygenase in the Developing Neonate

\begin{tabular}{llllll}
\hline & \multicolumn{2}{l}{ Serum bilirubin } & & \multicolumn{2}{l}{ Hepatic heme oxygenase } \\
\cline { 2 - 3 } $\begin{array}{llllll}\text { Time } \\
\text { postnatally }\end{array}$ & Control & $\begin{array}{l}\text { Heme- } \\
\text { treated }\end{array}$ & & Control & Heme-treated \\
\hline$d$ & $m g / d l$ & $m g / d l$ & & \multicolumn{2}{l}{ nmol bilirubin $/ \mathrm{mg} / \mathrm{h}$} \\
7 & $0.32 \pm 0.02$ & $4.08 \pm 0.48$ & & $7.55 \pm 0.03$ & $18.90 \pm 2.18$ \\
14 & $0.20 \pm 0.02$ & $4.51 \pm 0.60$ & & $5.36 \pm 0.98$ & $18.03 \pm 0.87$ \\
21 & $0.31 \pm 0.02$ & $2.16 \pm 0.05$ & & $3.15 \pm 0.07$ & $23.57 \pm 0.57$ \\
28 & $0.19 \pm 0.01$ & $0.48 \pm 0.04$ & & $2.38 \pm 0.21$ & $20.68 \pm 1.56$ \\
\hline
\end{tabular}

Heme $(10 \mu \mathrm{mol} / 100 \mathrm{~g} \mathrm{bw})$ administered subcutaneously 24 and $16 \mathrm{~h}$ prior to sacrifice. Control administered an equal volume of saline. Data are expressed as the mean $\pm S E$
4. Serum bilirubin levels had approximately doubled by $4 \mathrm{~h}$ and were substantially increased at 8,16 , and $24 \mathrm{~h}$ after the initial dose before returning to normal levels by $48-72 \mathrm{~h}$ in the treated animals (Fig. 4). Hepatic heme oxygenase activity did not significantly increase until $16 \mathrm{~h}$; enzyme activity reached a maximum $24 \mathrm{~h}$ after the initial administration of ALA before gradually declining towards control levels, which still had not been attained by $72 \mathrm{~h}$. The $24-\mathrm{h}$ time point was selected for further study because serum bilirubin levels were markedly elevated at this time, hepatic heme oxygenase was maximally increased, and the $12 / 12 \mathrm{~h}$ light/dark cycle under which the animals were maintained would not be disrupted.

Effect of Sn-protoporphyrin in vivo on ALA-induced hyperbilirubinemia and on the conversion of $\left[{ }^{14} C\right] A L A$ to heme in 7 $d$-old neonates. ALA $(50 \mu \mathrm{mol} / 100 \mathrm{~g} \mathrm{bw})$ was administered to suckling neonates 24,20 , and $16 \mathrm{~h}$ prior to sacrifice; Sn-protoporphyrin over the dose range of $10-100 \mu \mathrm{mol} / \mathrm{kg}$ bw was administered once at the time of the first dose of ALA. The metalloporphyrin administration produced a dose-dependent decrease in serum bilirubin levels (Fig. 5) with the highest dose of Sn-protoporphyrin diminishing the ALA-induced elevation in serum bilirubin levels by $\sim 60 \%$. The metalloporphyrin also produced a dose-dependent decrease in hepatic and splenic heme oxygenase activities (results not shown).

To insure that Sn-protoporphyrin did not lower serum bilirubin levels by interfering with the production of heme from ALA, and thus, providing less substrate for catabolism to bile pigment, the effect of Sn-protoporphyrin $(100 \mu \mathrm{mol} / \mathrm{kg} \mathrm{bw}) \mathrm{ad}$ ministration to suckling neonates on the conversion of $\left[{ }^{14} \mathrm{C}\right] \mathrm{ALA}$ to heme in liver was examined (Fig. 6). Sn-protoporphyrin administration did not reduce the formation of hepatic heme from $\left[{ }^{14} \mathrm{C}\right] \mathrm{ALA}$ through $24 \mathrm{~h}$; indeed a slight but significant increase in heme production at $4 \mathrm{~h}$ and at $24 \mathrm{~h}$ was noted (Fig. 6) after in vivo administration of the synthetic heme compound. The mechanism of the latter effect is not known.

Comparative effect of $\mathrm{Sn}$-, $\mathrm{Zn}$-, and Mn-protoporphyrin on ALA-induced hyperbilirubinemia and on hepatic heme oxygenase

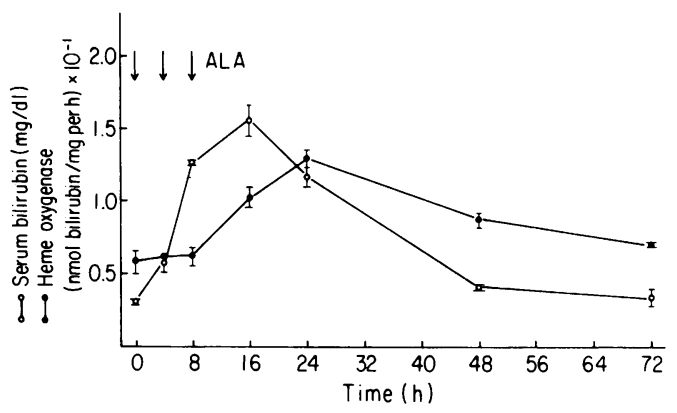

Figure 4. Effect of ALA on serum bilirubin levels and hepatic heme oxygenase activity in $7-\mathrm{d}$-old neonates. ALA $(50 \mu \mathrm{mol} / 100 \mathrm{~g} \mathrm{bw})$ was administered intraperitoneally at the times indicated to neonates. Animals were sacrificed at various times and hepatic heme oxygenase activity and serum bilirubin levels were assayed as described in Methods. A minimum of 24 neonates were used at each time point. 


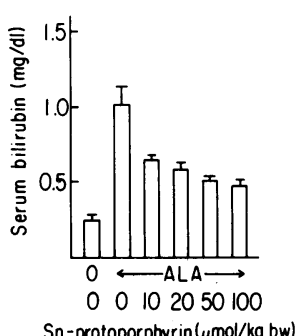

Sn-protoporphyrin( $\mu \mathrm{mol} / \mathrm{kg}$ bw)

Figure 5. Dose response effect of Sn-protoporphyrin on ALA-induced hyperbilirubinemia in 7-d-old neonates. ALA (50 $\mu \mathrm{mol} / 100 \mathrm{~g} \mathrm{bw}$ ) was administered intraperitoneally to suckling neonates in three separate doses 24,20 , and $16 \mathrm{~h}$ prior to sacrifice. Sn-protoporphyrin was administered subcutaneously in the doses indicated $24 \mathrm{~h}$ prior to sacrifice. Serum bilirubin levels were determined by the method of Roth (10). A minimum of 24 neonates were used in each group.

activity in 7-d-old neonates. ALA $(50 \mu \mathrm{mol} / 100 \mathrm{~g} \mathrm{bw})$ administered to suckling neonates at 0,4 , and $8 \mathrm{~h}$ produced an $\sim$ fivefold increase in serum bilirubin levels at $24 \mathrm{~h}$; thereafter, serum bilirubin decreased to essentially normal levels by $48 \mathrm{~h}$ (Fig. 7). Of the three metalloporphyrins examined in a single dose of $50 \mu \mathrm{mol} / \mathrm{kg}$ bw administered at 0 time, Sn-protoporphyrin substantially diminished the marked increase in serum bilirubin produced by ALA (Fig. 7); neither Mn- nor Zn-protoporphyrin in the same dose was able to suppress this induced hyperbilirubinemia (Tabie III). In addition, Sn-protoporphyrin administration prevented the increase in hepatic heme oxygenase activity associated with ALA administration (Fig. 7) and also substantially lowered splenic heme oxygenase activity (results not shown). $\mathrm{Mn}$ - and $\mathrm{Zn}$-protoporphyrin were largely ineffective in preventing the increase in hepatic heme oxygenase activity produced by ALA administration (Table III).

Sn-protoporphyrin ( $50 \mu \mathrm{mol} / \mathrm{kg} \mathrm{bw})$ administered to suckling neonates treated with heme $(10 \mu \mathrm{mol} / 100 \mathrm{~g} \mathrm{bw})$ at 24 and 16 $\mathrm{h}$ prior to sacrifice also significantly reduced the increase in serum bilirubin produced by heme (Table IV). Hepatic heme oxygenase activity was also reduced in the Sn-protoporphyrintreated group compared with the activity of this enzyme in the group treated with heme alone. Thus, Sn-protoporphyrin diminished the hyperbilirubinemia and the increase in heme oxygenase activity produced in the 7-d-old neonate by heme as well as that produced by the heme precursor, ALA.

\section{Discussion}

The purpose of the present investigation was to develop an experimental model of postnatal jaundice in the rat in order to facilitate studies of the ability of synthetic metalloporphyrins

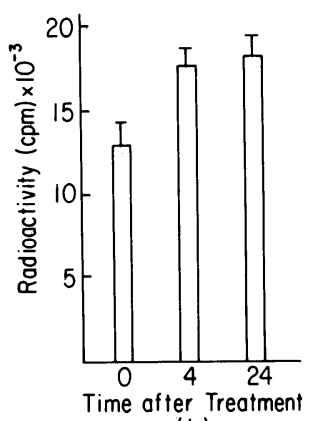

(h)
Figure 6. Effect of Sn-protoporphyrin on the in vivo conversion of $\left[{ }^{14} \mathrm{C}\right] \mathrm{ALA}$ to heme in liver in 7-d-old neonates. Snprotoporphyrin $(100 \mu \mathrm{mol} / \mathrm{kg} \mathrm{bw})$ was administered subcutaneously and animals sacrificed at the times indicated. The converison of $\left[{ }^{14} \mathrm{C}\right] \mathrm{ALA}$ to heme was determined by the method of Healty et al. (11). A minimum of 24 neonates were used at each time point.

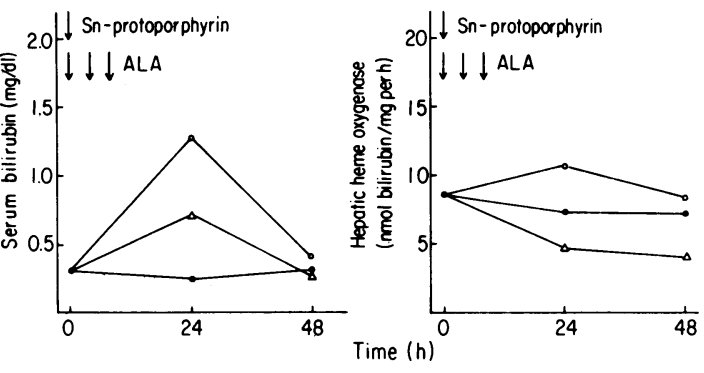

Figure 7. Effect of Sn-protoporphyrin on ALA-induced hyperbilirubinemia and hepatic heme oxygenase activity in 7-d-old neonates. ALA $(50 \mu \mathrm{mol} / 100 \mathrm{~g} \mathrm{bw})$ and Sn-protoporphyrin $(50 \mu \mathrm{mol} / \mathrm{kg} \mathrm{bw})$ were administered intraperitoneally and subcutaneously, respectively, at the times indicated. Animals were sacrificed at the times indicated and heme oxygenase activity and serum bilirubin levels were assayed as described in Methods. Control, $\bullet$; ALA-treated, O; and ALA- and Sn-protoporphyrin-treated, $\triangle$. Mean values are shown; other statistical data for this experiment are listed in Table III.

to suppress induced hyperbilirubinemia in this convenient laboratory animal species. The model that has been developed takes advantage of the fact that in the period after the immediate perturbations of heme metabolism associated with birth have subsided (up to $\sim 7 \mathrm{~d}$ ) but before the maturation of the hepatic glucuronyltransferase system for bilirubin is complete $(\sim 21 \mathrm{~d})$, it is possible to produce a consistent and significant hyperbilirubinemia in the rat by the administration of the heme precursor, ALA, or by heme itself. The use of ALA, in the present study, to produce hyperbilirubinemia in suckling neonates permitted the animals to remain with their mothers throughout the period of study so that they suckled in a normal manner. We believe this is an advantageous aspect of the use of ALA to produce jaundice. The ALA effect can be considerably enhanced by starvation (13); indeed, the exaggerated hyperbilirubinemic response to heme probably reflects the adverse effect of this compound on the feeding behavior of the animals. In

Table III. Effect of Zn-, Mn- and Sn-Protoporphyrin on ALA-induced Hyperbilirubinemia and Hepatic Heme Oxygenase Activity in the 7-d-old Neonate

\begin{tabular}{lll}
\hline Treatment & $\begin{array}{l}\text { Serum } \\
\text { bilirubin }\end{array}$ & $\begin{array}{l}\text { Hepatic heme } \\
\text { oxygenase }\end{array}$ \\
\hline & $m g / d l$ & nmol bilirubin/mg/h \\
Saline & $0.24 \pm 0.01$ & $7.25 \pm 0.10$ \\
ALA & $1.26 \pm 0.13$ & $10.60 \pm 0.73$ \\
ALA + Zn-protoporphyrin & $1.15 \pm 0.11$ & $12.60 \pm 1.26$ \\
ALA + Mn-protoporphyrin & $1.12 \pm 0.10$ & $9.00 \pm 0.24$ \\
ALA + Sn-protoporphyrin & $0.71 \pm 0.10^{*}$ & $4.68 \pm 0.24^{*}$
\end{tabular}

ALA $(3 \times 50 \mu \mathrm{mol} / 100 \mathrm{~g} \mathrm{bw})$ administered intraperitoneally 24,20 , and $16 \mathrm{~h}$ prior to sacrifice. Metalloporphyrin $(50 \mu \mathrm{mol} / \mathrm{kg} \mathrm{bw}) \mathrm{ad}-$ ministered subcutaneously $24 \mathrm{~h}$ prior to sacrifice. Data are expressed as the mean $\pm \mathrm{SE}$.

* $P<0.005$ compared with ALA-treated animals. 
Table IV. Effect of Sn-Protoporphyrin on

Heme-induced Hyperbilirubinemia and Hepatic Heme

Oxygenase Activity in 7-d-old Neonates

\begin{tabular}{llc}
\hline Treatment & $\begin{array}{l}\text { Serum } \\
\text { bilirubin }\end{array}$ & $\begin{array}{l}\text { Hepatic heme } \\
\text { oxygenase }\end{array}$ \\
\hline & $m g / d l$ & nmol bilirubin $/ \mathrm{mg} / \mathrm{h}$ \\
Saline & $0.28 \pm 0.03$ & $7.31 \pm 1.77$ \\
Heme & $4.08 \pm 0.48$ & $18.90 \pm 2.18$ \\
Heme + Sn-protoporphyrin & $2.88 \pm 0.04^{*}$ & $8.85 \pm 1.71^{*}$ \\
\hline
\end{tabular}

Heme administered subcutaneously in two doses of $10 \mu \mathrm{mol} / 100 \mathrm{~g}$ bw 24 and $16 \mathrm{~h}$ prior to sacrifice. Sn-protoporphyrin administered subcutaneously in a single dose of $50 \mu \mathrm{mol} / \mathrm{kg}$ bw $24 \mathrm{~h}$ prior to sacrifice. Data are expressed as the mean $\pm \mathrm{SE}$ of a minimum of three determinations.

${ }^{*} P<0.005$ compared with heme-treated animals.

addition, the normal $12 / 12 \mathrm{~h}$ light/dark cycle was not interrupted. Thus, insofar as was possible, a normal developmental and behavioral pattern was maintained in the neonates throughout the period of study of ALA-induced hyperbilirubinemia.

ALA administration produced a rapid, consistent, and significant increase in serum bilirubin levels in 7-14-d-old neonates (Fig. 3); this effect could not be elicited in animals 21 -d-old or older. Associated with the increase in serum bilirubin was a considerable increase in hepatic heme oxygenase activity (Fig. $3,4)$, presumably due to increased availability of ALA-derived heme. Administration of heme $(18,19)$ or ALA $(20)$ has been demonstrated to induce the enzyme in liver and other tissues of the adult animal. No effect of administered ALA was observed on the normal developmental pattern of splenic heme oxygenase activity in neonates. Thus, the elevated levels of serum bilirubin that occur after ALA administration in 7-14-d-old neonates can be considered to be due, in part, to an increase in the rate of heme oxidation to bile pigment in the liver, coupled with a limited capacity of the still not fully matured hepatic glucuronyltransferase system to conjugate the increased amounts of bile pigment formed. The fact that, after ALA administration, plasma bilirubin levels increased substantially before the induced increase in hepatic heme oxygenase was observed also suggests that heme oxygenase in spleen, and presumably other tissues including liver, is not substrate-saturated during the postnatal time period in which ALA loading was studied.

In the 7-14-d-old neonates examined in this study, the hepatic glucuronyltransferase system, while not fully matured, still appears capable of conjugating the normal amounts of bilirubin being formed in vivo at this time, since the immediate postnatal surge of hyperbilirubinemia is known to subside by $\sim 4 \mathrm{~d}$ after birth (2). ALA administration, however, leads to a consistent and substantial increase in serum bilirubin levels during the period 7-14 d postnatally, suggesting that the hepatic glucuronyltransferase system during this time period may be functioning at or close to maximum efficiency and that a substantial increase in bilirubin production in the liver and other tissues will quickly (within $4 \mathrm{~h}$ [Fig. 4]) saturate the conjugating mechanism for the bile pigment. At $\sim 21 \mathrm{~d}$ postnatally, it has been demonstrated that the glucuronyltransferase system for bilirubin in the liver is functioning at near-to-normal adult levels (16, 17). At this time, no difference in serum bilirubin levels was observed in this study between control and ALA-treated animals (Fig. 3), despite the marked increase in hepatic heme oxygenase activity which had been induced in the latter group. Similarly, neither ALA nor heme treatment produced significant hyperbilirubinemia in adult animals (Table I). The ability of ALA and of heme to produce hyperbilirubinemia in 7-14-d-old rats thus reflects a number of factors among which are the relative contributions of splenic and hepatic heme oxygenase to bile pigment formation, the extent to which the hepatic enzyme activity is induced by ALA or heme administration, and the maturity of the hepatic glucuronyltransferase for bilirubin within this time period.

The ability of Sn-protoporphyrin to suppress ALA-induced hyperbilirubinemia was consistent, substantial, and dose-dependent. In addition, this synthetic metalloporphyrin did not block the conversion in vivo of $\left[{ }^{14} \mathrm{C}\right] \mathrm{ALA}$ to heme in the liver, indicating that the markedly reduced levels of serum bilirubin observed after combined treatment of neonates with ALA and Sn-protoporphyrin were not due to reduced formation of heme from ALA.

In other studies, we have shown that Sn-protoporphyrin inhibits the marked increase in biliary bilirubin output, which is produced by infusion of exogenous heme or heat-damaged erythrocytes, and that the metalloporphyrin does not significantly alter the binding of bilirubin to purified human serum albumin or the hepatic uptake and biliary excretion of the infused bile pigment itself. Sn-protoporphyrin administration also produces a rapid (within 30-60 min) complete saturation of hepatic tryptophan pyrrolase which subsides after several hours and a substantial excretion of heme into bile occurs during this time period (manuscript in preparation). Thus, the Sn-protoporphyrin effect described in this study can be reasonably attributed to the ability of this compound to act as a potent and sustained inhibitor of heme oxygenase in vivo. This postulated mechanism does not exclude additional modes of action of the metalloporphyrin; these possibilities have been discussed in a recent report (21) and will not be reviewed here. It should be emphasized again $(2,3)$, however, that the level of heme oxygenase activity as measured in tissues in vitro does not necessarily equate with the rate of heme degradation in vivo (22) or with the ability of a competitive inhibitor of heme oxygenase to suppress hyperbilirubinemia $(2,3,23)$. Pharmacokinetic parameters and other biologic variables in vivo clearly influence these responses to synthetic metalloporphyrins and whole animal studies are thus essential in evaluating the potential ability of such compounds to suppress jaundice in the neonate.

Heme administration to suckling neonates during the period 7-21 d after birth resulted in significantly higher levels of serum bilirubin and hepatic heme oxygenase activity when compared with these parameters in neonates administered an $\sim$ eightfold molar excess of ALA (Table II). This may be due in part to the 
fact that preformed heme may be more quickly available for oxidative degradation and in part to an incomplete conversion of ALA to heme or to loss of ALA through excretion (20). The administration of heme, however, while producing a greater degree of hyperbilirubinemia than ALA also appeared to adversely affect the normal behavior and suckling patterns of the treated neonates. The latter effect would in its own right lead to an exaggerated hyperbilirubinemic response (13). Thus, ALA administration was favored as the method of choice for producing hyperbilirubinemia in this study. However, heme administration can provide, during the appropriate postnatal time period (i.e., 7-14 d), a means of acutely and markedly increasing the rate of endogenous bilirubin production when serum levels of this bile pigment greatly exceeding normal may be experimentally useful-as for example, in studies of the mechanism of the encephalopathy produced by the bile pigment in the newborn (24-26).

Sn-protoporphyrin is the most potent competitive inhibitor of heme oxygenase which we have yet identified and when administered either to rats $(2,3)$ or to nonhuman primates $(6,7)$, including Rhesus newborn (7), is effective in suppressing neonatal hyperbilirubinemia. This heme analogue also inhibits the markedly increased heme oxygenase activity which can be induced by metals or by metalloporphyrins, such as Co-protoporphyrin (27). In addition to its effects in animal neonates, Sn-protoporphyrin has proved effective in suppressing jaundice in mutant mice with severe hemolytic anemias (5), and as shown in this study, in postnatal rats in whom hyperbilirubinemia has been induced experimentally. The metalloporphyrin also suppresses to a considerable degree $(\sim 50 \%)$ the extreme hyperbilirubinemia which rapidly ensues after complete ligation of the bile duct in rats (manuscript in preparation); this form of jaundice may reflect in part the enhanced rate of hepatic heme oxidation which the experimental manipulation produces (28).

These studies, thus, confirm in other model systems of animal jaundice the findings we first reported in 1981 (2) concerning the potent ability of Sn-protoporphyrin to suppress hyperbilirubinemia in situations in which exaggerated rates of heme degradation occur, as in the neonate $(9,14)$. The pharmacokinetic properties of Sn-protoporphyrin have now been defined in the rat (29), but comparable data on the pharmacological properties or toxicity, if any, of the compound in man are not yet available. Studies along these lines are in progress in this laboratory. Initial (21) findings indicate that the ability of this synthetic metalloporphyrin to diminish excessive levels of serum bilirubin in experimental animals extends to humans as well.

\section{Acknowledgments}

We thank Ms. Susan Spinner for her technical assistance and Mrs. Heidemarie Robinson for the preparation of this manuscript.

This study was supported by a grant from the Dyson Foundation, NY, grant 1-804 from the March of Dimes Birth Defects Foundation, and by U. S. Public Health Service grant ES-01055.

\section{References}

1. Gartner, L. M. 1982. Hyperbilirubinemia. In Pediatrics. A. M. Rudolph, editor. Appleton-Century-Crofts, New York. Seventeenth ed. 1007-1013.

2. Drummond, G. S., and A. Kappas. 1981. Prevention of neonatal hyperbilirubinemia by tin-protoporphyrin IX, a potent competitive inhibitor of heme oxidation. Proc. Natl. Acad. Sci. USA. 78:6466-6470.

3. Drummond, G. S., and A. Kappas. 1982. Chemoprevention of neonatal jaundice: potency of tin-protoporphyrin in an animal model. Science (Wash. DC). 217:1250-1252.

4. Yoshinaga, T., S. Sassa, and A. Kappas. 1982. Purification and properties of bovine spleen heme oxygenase: amino acid composition and sites of action of inhibitors of heme oxidation. J. Biol. Chem. 257:7778-7785.

5. Sassa, S., G. S. Drummond, S. E. Betnstein, and A. Kappas. 1983. Tin-protoporphyrin suppression of hyperbilirubinemia in mutant mice with severe hemolytic anemia. Blood. 61:1011-1013.

6. Chowdhury, J. R., O. W. Portman, N. R. Chowdhury, M. Alexander, C. E. Cornelius, and I. M. Arias. 1982. Mechanism of fasting hyperbilirubinemia studies in Bolivian Squirrel Monkeys. Hepatology (Baltimore). 2:723.

7. Rodgers, P. A., M. L. Bruss, and C. E. Cornelius. 1983. Further characterization of a Gilbert-like syndrome in Bolivian Squirrel Monkeys (Saimiri Squeurus). Fed. Proc. 42:1256.

8. Drummond, G. S., and A. Kappas. 1982. Suppression of hyperbilirubinemia in the rat neonate by chromium protoporphyrin: interactions of metalloporphyrins with microsomal heme oxygenase of human spleen. J. Exp. Med. 156:1878-1883.

9. Maines, M. D., and A. Kappas. 1975. Study of the developmental patterns of heme catabolism in liver and the effects of cobalt on cytochrome $\mathrm{P}-450$ and the rate of heme oxidation during the neonatal period. J. Exp. Med. 141:1400-1410.

10. Roth, M. 1967. Dosage fluorimetrique de la bilirubine. Clin. Chim. Acta. 17:487-492.

11. Healey, J. F., H. L. Bonkowsky, P. R. Sinclair, and J. F. Sinclair. 1981. Conversion of $\delta$-aminolaevulinate into haem by liver homogenates; comparison of rat and chick embyro. Biochem. J. 198:595-604.

12. Lowry, O., N. J. Rosebrough, A. L. Farr, and R. J. Randall. 1951. Protein measurement with the folin phenol reagent. J. Biol. Chem. 193:265-275.

13. Song, C. S., H. L. Moses, A. S. Rosenthal, N. A. Gelb, and A Kappas. 1971. The influence of postnatal development on drug-induced hepatic porphyria and the synthesis of cytochrome P-450: a biochemical and morphological study. J. Exp. Med. 134:1349-1371.

14. Thaler, M. M., D. L. Gemes, and A. F. Bakken. 1972. Enzymatic conversion of heme to bilirubin in normal and starved fetuses and newborn rats. Pediatr. Res. 6:197-201.

15. Maines, M. D. 1981. Zinc-protoporphyrin is a selective inhibitor of heme oxygenase activity in the neonatal rat. Biochim. Biophys. Acta. 673:339-350.

16. Halac, E., Jr., and C. Sicignano. 1969. Re-evaluation of the influence of sex, age, pregnancy, and phenobarbital on the activity of UDP-glucuronyl transferase in rat liver. J. Lab. Clin. Med. 73:677-685.

17. Strebel, L., and G. B. Odell. 1971. Bilirubin uridine diphosphoglucuronyltransferase in rat liver microsomes: genetic variation and maturation. Pediatr. Res. 5:548-559.

18. Tenhunen, R., H. S. Marver, and R. Schmid. 1970. The enzymatic catabolism of hemoglobin: stimulation of microsomal heme oxygenase by hemin. J. Lab. Clin. Med. 75:410-421. 
19. Pimstone, M. R., P. Engel, R. Tenhunen, P. T. Seitz, H. S. Marver, and R. Schmid. 1971. Inducible heme oxygenase in the kidney: a model for the homeostatic control of hemoglobin catabolism. J. Clin. Invest. 50:2024-2050.

20. Anderson, K. E., G. S. Drummond, U. Freddara, M. K. Sardana, and S. Sassa. 1981. Porphyrogenic effects and induction of heme oxygenase in vivo by $\delta$-aminolevulinic acid. Biochim. Biophys. Acta. 676:289-299.

21. Kappas, A., G. S. Drummond, C. S. Simionatto, and K. E. Anderson. 1984. Control of heme oxygenase and plasma levels of bilirubin by a synthetic heme analogue, tin-protoporphyrin. Hepatology (Baltimore). 4:336-341.

22. Drummond, G. S., D. W. Rosenberg, and A. Kappas. 1982. Metal induction of haem oxygenase without concurrent degradation of cytochrome P450: protective effects of SKF525A on the haem protein. Biochem. J. 202:59-66.

23. Kappas, A., and G. S. Drummond. 1982. The regulation of heme oxidation by synthetic metalloporphyrins: experimental and clinical implications. In Microsomes, Drug Oxidations, and Drug Toxicity. Proc. of the Fifth International Symposium on Microsomes and Drug Oxi- dations, Tokyo 1981. Japan Scientific Societies Press, Tokyo/WileyInterscience, New York. 629-636.

24. Turkel, S. B., C. A. Miller, M. E. Guttenberg, D. R. Maynes, and J. E. Hodgman. 1982. A clinical pathologic reappraisal of Kernicterus. Pediatrics. 69:267-272.

25. Levine, R. L., W. R. Fredericks, and S. I. Rapoport. 1982. Entry of bilirubin into the brain due to opening of the blood-brain barrier. Pediatrics. 69:255-259.

26. Brodersen, R. 1980. Bilirubin transport in the newborn infant, reviewed with relationship to kernicterus. J. Pediatr. 96:349-356.

27. Kappas, A., and G. S. Drummond. 1984. Control of heme and cytochrome P-450 metabolism by inorganic metals, organometals and synthetic metalloporphyrins. Environ. Health Perspect. In press.

28. Schacter, B. A., E. Joseph, and G. Firneisz. 1983. Effect of cholestasis produced by bile duct ligation on hepatic heme and hemoprotein metabolism in rats. Gastroenterology. 84:227-235.

29. Anderson, K. E., C. S. Simionatto, G. S. Drummond, and A. Kappas. 1984. Tissue distribution and disposition of tin-protoporphyrin, a potent competitive inhibitor of heme oxygenase. J. Pharmacol. Exp. Ther. 228:327-333. 